\title{
Verifying Extra-Pulmonary Manifestation of COVID- 19 in Firoozgar Hospital 2020: An Observational Study
}

\section{Azadeh Mottaghi}

IUMS: Iran University of Medical Sciences

\section{Maryam Roham}

IUMS: Iran University of Medical Sciences

Mahin Jamshidi Makiani

IUMS: Iran University of Medical Sciences

\section{Mitra Ranjbar}

IUMS: Iran University of Medical Sciences

\section{Azadeh Laali}

IUMS: Iran University of Medical Sciences

Neda Rahimian Rahimian.n@iums.ac.ir ( $\square$ Rahimian.n@iums.ac.ir)

Iran University of Medical Sciences https://orcid.org/0000-0002-1325-8946

\section{Research article}

Keywords: COVID-19, corona virus, extra-pulmonary manifestations

Posted Date: January 19th, 2021

DOI: https://doi.org/10.21203/rs.3.rs-147631/v1

License: (1) This work is licensed under a Creative Commons Attribution 4.0 International License. Read Full License 


\section{Abstract}

Background: COVID-19 is a new emerging disease that causes a pandemic situation in the world. Corona virus can enter into the body via several ways and it damages other organs of the body in addition to the respiratory system. This study aimed at verifying extra-pulmonary manifestation of COVID-19.

Methods: The present study was done as cross-sectional in the single center from March 1 to May 12020 at Firoozgar educational hospital in Tehran, Iran. 107 patients with confirmed Covid-19 pneumonia according to $\mathrm{WHO}$ interim guidance were recruited in this study. Extra-pulmonary manifestations of COVID-19 were recorded. SPSS, version 26 was used for all analyses.

Results: The mean (SD) and median of age was 59.3 (17.4) and 62.0 respectively and $58(54.2 \%)$ were men. Body temperature of patients who were equal or less than 60 years was significantly higher than other patients (39.02 vs. $38.08, \mathrm{p}=0001$ ). The most common extra-pulmonary manifestation was $\mathrm{GI}$ symptoms included nausea, vomiting, abdominal pain, diarrhea, Hepatocellular LFT abnormality, Cholestatic LFT abnormality and Amylase lipase incensement (37 patients (34.6\%)). Ophthalmological, cardiac, neurological and dermatological manifestations were shown in $6.5 \%, 6.5 \%, 14.9 \%$ and $14.0 \%$ of patients respectively.

Conclusion: Investigating the clinical and radiological symptoms of COVID-19 showed that SARS-CoV-2 infection may also be associated with extrapulmonary symptoms. Therefore, clinicians and radiologists should be familiar with such symptoms of this disease.

\section{Introduction}

A new coronavirus causes COVID-19 infection. COVID-19 cases suffer from mild to moderate respiratory disease and they recovered with no special treatment. Older cases and people with underlying medical diseases, such as cardiovascular disease, diabetes, chronic respiratory disorder, and cancer are at higher risk for developing serious illnesses.

Respiratory symptoms have been investigated in this disease (1). Along with respiratory and other typical symptoms, like fever, cough and muscle ache, extra-pulmonary manifestations have been reported in some cases requiring special care and attention. Neurological (2), cardiac (3), gastrointestinal (GI) (4), dermatological (5) and ophthalmological (6) manifestations have been less evaluated, which should be considered more.

Coronaviruses (CoVs) are able to go into the CNS via the bloodstream or neuronal retrograde pathway and cause meningitis, encephalitis, related morbidity, and risk of death. It is usually hard to diagnose viral encephalitis due to subtle or lack of symptoms; however, it is possible to detect some symptoms of the serious viral encephalitis including changed mental status and body temperament, unusual behavior or speech, unusual motor movement and focal neurological dysfunctions, like flaccid paralysis, paresthesia, hemiparesis, or seizures (7). 
Gastrointestinal (GI) symptoms have been reported in several patients. Some COVID-19 patients with GI symptoms were found as the only manifestation of the disease with no respiratory disorders $(8,9)$. It has been reported that from 67 COVID-19 cases with diarrhea, 13 patients had no other symptoms (10). Although GI symptoms in COVID-19 are not specific, they are more common in cases with approved SARS-CoV-2 infection.

Cardiovascular symptoms of COVID-19 are also challenging for physicians. Identification of the damage by COVID-19 to the cardiovascular system and the underlying mechanisms is crucial, because treating these cases is timely and effective with reduced mortality (3).

Recent studies have verified skin symptoms of COVID-19. The results of evaluating 88 COVID-19 cases, skin symptoms are erythematous rash, widespread urticaria, and chickenpox-like vesicles. Trunk skin lesions as the main area affected were not somewhat itchy (5).

The transmission of COVID-19 is done through droplets, however, other methods of transmission have been documented, for instance, transmitting via infected ocular tissue or fluid $(11,12)$. The nasolacrimal system may pave the way for viruses to travel from the upper respiratory tract to the eyes. Consequently, ocular tissue and fluid are probably a likely source of coronavirus.

This study aimed at verifying extra-pulmonary manifestation of COVID-19.

\section{Methods}

The present study was done as cross-sectional in the single center from March 1 to May 12020 at Firoozgar educational hospital in Tehran, Iran. 107 patients with confirmed Covid-19 pneumonia according to $\mathrm{WHO}$ interim guidance were recruited in this study.

This study was approved by Ethics Committee of Iran University of Medical Sciences and all patients signed written informed consent. Two attending doctors separately examined patients for neurological, cardiac, GI, ophthalmological and dermatological manifestations. Lung CT involvement was categorized as mild, moderate and severs. Presence of cough, dyspnea, chest pain, anosmia and dysgeusia were recorded. Past medical history such as suffering from diabetes, hypertension (HTN), ischemic heart disease (IHD), chronic kidney disease (CKD), Immuno-compromised status, myalgia and other diseases were collected from all patients.

\section{Statistical analysis}

Continuous measurements reported as mean \pm Standard deviation (SD) for normally distributed data and categorical variables were described as number of patients (percent). SPSS, version 26 was used for all analyses. Continuous variables were compared using independent sample t-test and proportions for categorical variables were compared using the $\chi^{2}$ test, whereas the Fisher exact test was conducted when the data were limited. 


\section{Results}

The study participants included 107 hospitalized patients with confirmed Covid-19 pneumonia. Basic characteristics of the study participants according to age groups are illustrated in Table 1. The mean (SD) and median of age was $59.3(17.4)$ and 62.0 respectively and $58(54.2 \%)$ were men. Body temperature of patients who were equal or less than 60 years was significantly higher than other patients (39.02 vs. $38.08, p=0001)$. Of patients older than 60 years, $16(28.6 \%)$ and $55(98.2 \%)$ had HTN and dyspnea, respectively. Surprisingly, patients who were equal or less than 60 years, had significantly more underlying comorbid systemic disease than other patients (9 (17.3\%) vs. $2(3.6 \%), P=0.019)$. Figure.1 shows comorbidities of patients on admission. 
Table 1

Baseline characteristics of participants by age group

\begin{tabular}{|c|c|c|c|}
\hline Baseline characteristics & Age $\leq 60$ years $(n=51)$ & Age $>60$ years $(n=56)$ & p-value \\
\hline \multicolumn{4}{|l|}{ Continues variables ${ }^{*}$} \\
\hline Hospitalization duration & $9.53 \pm 7.06$ & $10.50 \pm 5.60$ & 0.431 \\
\hline Body temperature & $39.02 \pm 0.39$ & $38.08 \pm 0.51$ & 0.0001 \\
\hline \multicolumn{4}{|l|}{ Categorical variables $^{\star *}$} \\
\hline Sex & $24(49.0)$ & $25(51.0)$ & 0.848 \\
\hline Female & $27(46.6)$ & $31(53.4)$ & \\
\hline \multicolumn{4}{|l|}{ Male } \\
\hline Diabetes & $11(21.0)$ & $18(32.1)$ & 0.142 \\
\hline Ischemic heart disease & $7(13.5)$ & $14(25.0)$ & 0.101 \\
\hline Chronic kidney disease & $2(3.8)$ & $2(3.6)$ & 0.662 \\
\hline Hypertension & $3(5.8)$ & $16(28.6)$ & 0.002 \\
\hline Immuno-compromised status & $1(1.9)$ & $1(1.8)$ & 0.773 \\
\hline Other diseases & $9(17.3)$ & $2(3.6)$ & 0.019 \\
\hline Opium use & $1(1.9)$ & $1(1.8)$ & 0.733 \\
\hline Smoking & $7(13.5)$ & $7(12.5)$ & 0.554 \\
\hline Myalgia & $2(3.8)$ & $0(0.0)$ & \\
\hline Lung CT involvement & $11(21.2)$ & $11(19.6)$ & \\
\hline Mild & $19(36.5)$ & $26(46.4)$ & \\
\hline Moderate & $14(26.9)$ & $19(33.9)$ & \\
\hline \multicolumn{4}{|l|}{ severe } \\
\hline Cough & $46(88.5)$ & $52(92.9)$ & 0.325 \\
\hline Dyspnea & $44(86.3)$ & $55(98.2)$ & 0.022 \\
\hline Chest discomfort & $29(56.9)$ & $36(64.3)$ & 0.279 \\
\hline Anosmia & $8(15.7)$ & $4(7.1)$ & 0.137 \\
\hline \multicolumn{4}{|l|}{ *data reported as mean \pm SD } \\
\hline${ }^{* *}$ data reported as number of $p$ & ent (percent) & & \\
\hline
\end{tabular}




\begin{tabular}{|llll|}
\hline Baseline characteristics & Age $\leq 60$ years $(n=51)$ & Age $>60$ years $(n=56)$ & p-value \\
\hline dysgeusia & $8(15.7)$ & $3(5.4)$ & 0.075 \\
\hline${ }^{*}$ data reported as mean \pm SD & & & \\
\hline${ }^{* *}$ data reported as number of patient (percent) & & \\
\hline
\end{tabular}

Extra-pulmonary manifestations related to COVID-19 patients are shown in Table 2. The most common extra-pulmonary manifestation was GI symptoms included nausea, vomiting, abdominal pain, diarrhea, Hepatocellular LFT abnormality, Cholestatic LFT abnormality and Amylase lipase incensement (37 patients (34.6\%)). Ophthalmological (7 patients (6.5\%)) and cardiac (7 patients (6.5\%)) manifestations were the lowest clinical involvement related to Covid-19 pneumonia. Neurological manifestations consist of ischemic CVA, Intracerebral hemorrhage (ICH), Guillain-Barré syndrome (GBS) and encephalitis were seen in 16 patients (14.9\%). Of 107 patients who participated in the present study, 15 patients $(14.0 \%)$ showed dermatological symptoms such as erythema, macula, papule, morbid form rash, urticarial and patch (Figure.2). 
Table 2

Extra-pulmonary manifestation related to covid-19 patients

\begin{tabular}{|c|c|c|c|}
\hline Variables & Age $\leq 60$ years $(n=51)$ & Age $>60$ years $(n=56)$ & p-value \\
\hline Neurological manifestations & $10(19.6)$ & $6(10.7)$ & 0.155 \\
\hline Ischemic CVA & $0(0)$ & $2(3.6)$ & 0.496 \\
\hline $\mathrm{ICH}$ & $0(0)$ & $0(0)$ & - \\
\hline GBS & $1(2.0)$ & $0(0)$ & 0.477 \\
\hline Encephalitis & $2(3.9)$ & $0(0)$ & 0.225 \\
\hline Cardiac manifestations & $4(7.8)$ & $3(5.4)$ & 0.448 \\
\hline Myocardial infarction & $2(3.9)$ & $2(3.6)$ & 0.655 \\
\hline Pulmonary embolism & $0(0)$ & $1(1.8)$ & 0.523 \\
\hline Pericarditis & $1(2.0)$ & $1(1.8)$ & 0.728 \\
\hline Myocarditis & $1(2.0)$ & $1(1.8)$ & 0.728 \\
\hline GI manifestations & $21(41.2)$ & $16(28.6)$ & 0.122 \\
\hline Nausea, vomiting & $20(39.2)$ & $14(25.0)$ & 0.085 \\
\hline Abdominal pain & $14(27.5)$ & $12(21.4)$ & 0.308 \\
\hline Diarrhea & $11(21.6)$ & $8(14.3)$ & 0.232 \\
\hline Hepatocellular LFT abnormality & $13(25.5)$ & $10(17.9)$ & 0.506 \\
\hline Cholestatic LFT abnormality & $0(0)$ & $0(0)$ & - \\
\hline Amylase lipase incensement & $3(5.9)$ & $0(0)$ & 0.105 \\
\hline Ophthalmologic manifestations & $5(9.8)$ & $2(3.6)$ & 0.491 \\
\hline Ocular irritation & $4(7.8)$ & $1(1.8)$ & 0.154 \\
\hline Ocular itching & $4(7.8)$ & $1(1.8)$ & 0.154 \\
\hline Epiphora & $1(2.0)$ & $0(0)$ & 0.477 \\
\hline Foreign body sensation & $4(7.8)$ & $2(3.6)$ & 0.296 \\
\hline Ocular secretion & $1(2.0)$ & $1(1.8)$ & 0.728 \\
\hline Conjunctival hyperemia & $4(7.8)$ & $2(3.6)$ & 0.296 \\
\hline Shirmer left & $5(9.8)$ & $2(3.6)$ & 0.491 \\
\hline Shirmer right & $5(9.8)$ & $2(3.6)$ & 0.491 \\
\hline
\end{tabular}




\begin{tabular}{|c|c|c|c|}
\hline Variables & Age $\leq 60$ years $(n=51)$ & Age $>60$ years $(n=56)$ & p-value \\
\hline Tear or secretion PCR test & $0(0)$ & $0(0)$ & - \\
\hline Eye side involvement & $5(9.8)$ & $2(3.6)$ & 0.491 \\
\hline Dermatologic manifestations & $10(19.6)$ & $5(8.9)$ & 0.163 \\
\hline Erythema & $6(11.8)$ & $3(5.4)$ & 0.200 \\
\hline Macula & $4(7.8)$ & $2(3.6)$ & 0.296 \\
\hline Papule & $4(7.8)$ & $2(3.6)$ & 0.296 \\
\hline Patch & $3(5.9)$ & $0(0)$ & 0.105 \\
\hline Plaque & $2(3.9)$ & $1(1.8)$ & 0.604 \\
\hline Nodule & $0(0)$ & $0(0)$ & - \\
\hline Pustule & $0(0)$ & $0(0)$ & - \\
\hline Vesicle & $1(2.0)$ & $1(1.8)$ & 0.728 \\
\hline Urticarial & $3(5.9)$ & $1(1.8)$ & 0.274 \\
\hline Scaling & $2(3.9)$ & $1(1.8)$ & 0.604 \\
\hline Itching & $2(3.9)$ & $1(1.8)$ & 0.604 \\
\hline Irritation or pain & $1(2.0)$ & $2(3.6)$ & 1.000 \\
\hline Secretion PCR test & $0(0)$ & $0(0)$ & - \\
\hline Morbid form rash & $4(7.8)$ & $2(3.6)$ & 0.421 \\
\hline Petechial & $2(3.9)$ & $1(1.8)$ & 0.604 \\
\hline Acral syansis & $0(0)$ & $2(3.6)$ & 0.496 \\
\hline Livedoreticularis & $1(2.0)$ & $0(0)$ & 0.477 \\
\hline
\end{tabular}

\section{Discussion}

The current study focuses on the epidemiology and clinical characteristics of 107 COVID-19 cases referred to Firoozgar hospital. Human coronavirus is an important cause of respiratory tract infection. SARSCoV and MERSCoV lead to severe respiratory syndrome in humans, and HCoVOC43, HCoV229E, HCoVNL63, and HCoVHKU1 as other human coronaviruses cause mild upper respiratory disorder.

There was no significant difference between the number of male and female subjects. In many studies evaluating COVID-19 cases, the numbers of men were more than women $(1,13,14)$. The decreased 
vulnerability of women to viral infections may be associated with the protection from $\mathrm{X}$ chromosome and sex hormones that are crucial in innate and adaptive immunity (15). Approximately, $30 \%$ of cases affected by coronavirus were found with hypertension, diabetes and ischemic heart disease, which is also true for MERS-CoV.19 (13). Our findings indicated that younger cases had remarkably more underlying comorbid systemic disease compared to older cases, which can be associated with underlying diseases that weaken the immune system and increase the risk of infection with coronavirus (16).

GI symptoms are more prevalent among the extrapulmonary manifestations. Nausea, vomiting, abdominal pain, diarrhea, Hepatocellular liver function test abnormalities, cholestasis, and Amylase lipase incensement have been reported in nearly $45 \%$ of cases. Some cases of COVID-19 have been observed with GI symptoms as the only manifestation of the disease with no respiratory dysfunctions (9). GI wall permeability is enhanced to foreign pathogens while infected with the virus, GI symptoms, like diarrhea, are observed because of mal-absorption of infected enterocytes (4). Cohort studies have also consistently announced GI symptoms in Covid-19 cases. Another study on 1,099 patients from 552 hospitals in China reported nausea or vomiting in 55 (5.0\%) and diarrhea in 42 (3.8\%) patients (17). Many cohort studies have revealed diarrhea (2.0-10.1\%) and nausea and/or vomiting (1.0-10.1\%) in these patients $(1,18,19)$. Consistent with the current study, in a cohort study of 140 Covid-19 cases in Wuhan, up to $39.6 \%$ of them had GI symptoms, such as nausea $(24,17.3 \%)$, diarrhea $(18,12.9 \%)$, and vomiting ( 7 , $5.0 \%)(20)$. The receptor-binding domain on SARS-CoV-2 is capable of binding to human ACE2 with strong affinity, which is believed to help spread the virus among human population effectively $(21,22)$. Significant expression of ACE2 in alveolar cells type II (AT2) in the lungs has been reported, and the receptor can be also extensively expressed in the GI tract, chiefly in the small and large intestines (23). Staining of the virus nucleocapsid protein was observed in cytoplasm of gastric, duodenal, and rectal epithelium (24). Thus, it is possible to consider receptor-mediated entry into the host cells can be revealing a basis for its probable transmission way via the fecal contents.

Dermatological symptoms have been recorded in nearly $20 \%$ of 107 COVID-19 cases. Erythema has been the most common manifestation, particularly in the patients $\leq 60$ years. It has been reported that patients with confirmed SARS-CoV-2 infection might develop cutaneous involvement. In another study on 88 COVID-19 patients, it was observed that erythematous rash, widespread urticaria, and chickenpox-like vesicles are the skin symptoms of the COVID-19. Trunk skin lesions as the main area affected were not somewhat itchy (5).

CoVs are capable of going into the CNS via the bloodstream or neuronal retrograde route causing meningitis and encephalitis along with morbidity and risk of mortality. It is hard to diagnose viral encephalitis due to subtle or lack of symptoms, however, symptoms in severe viral encephalitis are changed mental status and body temperament, unusual behavior or speech, abnormality in motor movement and focal neurological dysfunctions, including flaccid paralysis, paresthesia, hemiparesis, or seizures (7). Assessing 214 patients with COVID-19 indicated that 78 patients (36.4\%) had neurological manifestations, like headache, dizziness, acute cerebrovascular diseases, and disturbed consciousness (25). 
Studies on the neurological attack in COVID-19 pneumonia revealed that SARSCoV-2 was capable of attacking the CNS and finding the way throughout the systemic circulation or through the cribriform plate of the ethmoid bone. It could then damage the neuronal tissues via interaction with ACE2 receptors. COVID-19 cerebral participation through the cribriform plate can cause more complications, like hyposmia or anosmia $(26,27)$. Some investigations have reported the likely cerebrovascular endothelial rupture leading to bleeding and irrepressible consequences (27). Due to intracerebral hemorrhage, cerebrovascular accidents have been declared as the rare COVID-19 neurological consequence (28). COVID-19-related acute hemorrhagic necrotizing encephalopathy was found in an individual with changed mental status with nasopharyngeal swab positive for SARS-CoV-2. According to imaging results, symmetric hypo attenuation in the bilateral medial thalami was detected by the head CT, as well as hemorrhagic ring-enhancing lesions in the bilateral thalami, medial temporal lobes, and sub insular areas detected by brain MRI (29). Nevertheless, more investigations should be done for the causation link.

Cardiac and ophthalmological symptoms have been reported in less than $10 \%$ of COVID-19 cases in our study. Of 107 studied patients, only 4 cases showed myocardial infarction. ACE1 is the target receptor for SARS-CoV-2 expressing remarkably in the heart. This transmembrane aminopeptidase is linked with developing hypertension, and heart function is essential in the cardiovascular system. Therefore, it is possible to consider cardiovascular injury or myocarditis a COVID-19 manifestation (30). Zheng et al. studied 5 COVID-19 cases with myocardial injury through the course of the disease. Myocardial injury is typically manifested as an elevated level of biochemical markers, such as cardiac troponin I (cTnl), creatine kinase (CK), a-hydroxybutyrate dehydrogenase (HBDB), and lactate dehydrogenase (LDH) (31). The impact of COVID-19 on acute myocardial infarction (MI), such as STEMI and NSTEMI was reported in the new protocols of acute MI (32).

According to the American Academy of Ophthalmology report, conjunctival infection and chemosis are some extrapulmonary symptoms of the infection with SARS-CoV-2 (33). Transmitting by infected ocular tissue or fluid should be more studied and is debated $(11,12)$. Nasolacrimal system may pave the way for the viruses to travel from the upper respiratory tract to the eyes. Consequently, it is possible to consider ocular tissue and fluid as likely source of SARS-CoV-2. However, in the present study, tear or secretion PCR test for detecting coronavirus was not positive.

\section{Conclusion}

COVID-19 leads to viral pneumonia with some clinical symptoms. According to the clinical and radiological symptoms of COVID-19, SARS-CoV-2 infection might also be related with extrapulmonary symptoms. Consequently, clinicians and radiologists are advised to be aware of such symptoms of this disease. It is essential to perform more studies to verify the causal link between SARS-CoV-2 infection and the reported extrapulmonary symptoms of COVID-19.

\section{Abbreviations}


Coronaviruses (CoVs)

Gastrointestinal (GI)

Hypertension (HTN)

Ischemic heart disease (IHD)

Chronic kidney disease (CKD)

Standard deviation (SD)

Intracerebral hemorrhage (ICH)

Guillain-Barré syndrome (GBS)

Alveolar cells type II (AT2)

Cardiac troponin I (cTnl)

Creatine kinase (CK)

Hydroxybutyrate dehydrogenase (HBDB)

Lactate dehydrogenase (LDH)

Myocardial infarction (MI)

\section{Declarations}

Acknowledgements

N/A

Authors' contributions

MR and NR designed the project. AM and AL collected the data and analyzed data. AM wrote the manuscript. MR and MJM supervised project and critically revised the written manuscript. All authors read, made significant edits to the first version, and approved the final manuscript.

\section{Funding}

The present study was supported by Iran University of Medical Sciences (grant no. IUMS16725): They had no role in the design of the study and collection, analysis, and interpretation of data and in writing the manuscript. 
The datasets used and/or analyzed in the present study are available from the corresponding author upon reasonable request.

\section{Ethics approval and consent to participate}

This study was approved by the Ethics Committee of Iran University of Medical Sciences. Written informed consent was obtained from all the participants.

Consent for publication

$\mathrm{N} / \mathrm{A}$

\section{Competing interests}

All authors declare no competing interests.

\section{References}

1. Chen N, Zhou M, Dong X, Qu J, Gong F, Han Y, et al. Epidemiological and clinical characteristics of 99 cases of 2019 novel coronavirus pneumonia in Wuhan, China: a descriptive study. The Lancet. 2020;395(10223):507-13.

2. Bridwell R, Long B, Gottlieb M. Neurologic complications of COVID-19. Am J Emerg Med. 2020.

3. Zheng Y-Y, Ma Y-T, Zhang J-Y, Xie X. COVID-19 and the cardiovascular system. Nature Reviews Cardiology. 2020;17(5):259-60.

4. Gu J, Han B, Wang J. COVID-19: gastrointestinal manifestations and potential fecal-oral transmission. Gastroenterology. 2020;158(6):1518-9.

5. Recalcati S. Cutaneous manifestations in COVID-19: a first perspective. Journal of the European Academy of Dermatology and Venereology. 2020.

6. Romano MR, Montericcio A, Montalbano C, Raimondi R, Allegrini D, Ricciardelli G, et al. Facing COVID-19 in Ophthalmology department. Current Eye Research. 2020(just-accepted).

7. Desforges M, Le Coupanec A, Dubeau P, Bourgouin A, Lajoie L, Dubé M, et al. Human Coronaviruses and Other Respiratory Viruses: Underestimated Opportunistic Pathogens of the Central Nervous System? Viruses. 2020;12(1):14.

8. Corley DA, Peek RM. COVID-19: What Should Clinicians and Scientists Do and When? Gastroenterology. 2020.

9. Pan L, Mu M, Yang P, Sun Y, Wang R, Yan J, et al. Clinical characteristics of COVID-19 patients with digestive symptoms in Hubei, China: a descriptive, cross-sectional, multicenter study. The American journal of gastroenterology. 2020;115.

10. Han C, Duan C, Zhang S, Spiegel B, Shi H, Wang W, et al. Digestive symptoms in COVID-19 patients with mild disease severity: clinical presentation, stool viral RNA testing, and outcomes. The American Journal of Gastroenterology. 2020. 
11. Lu C-w, Liu X-f, Jia Z-f. 2019-nCoV transmission through the ocular surface must not be ignored. Lancet (London, England). 2020;395(10224):e39.

12. Seah I, Agrawal R. Can the coronavirus disease 2019 (COVID-19) affect the eyes? A review of coronaviruses and ocular implications in humans and animals. Ocul Immunol Inflamm. 2020;28(3):391-5.

13. Badawi A, Ryoo SG. Prevalence of comorbidities in the Middle East respiratory syndrome coronavirus (MERS-CoV): a systematic review and meta-analysis. International Journal of Infectious Diseases. 2016;49:129-33.

14. Channappanavar R, Fett C, Mack M, Ten Eyck PP, Meyerholz DK, Perlman S. Sex-based differences in susceptibility to severe acute respiratory syndrome coronavirus infection. J Immunol. 2017;198(10):4046-53.

15. Jaillon S, Berthenet K, Garlanda C. Sexual dimorphism in innate immunity. Clinical reviews in allergy \& immunology. 2017:1-14.

16. Dryden M, Baguneid M, Eckmann C, Corman S, Stephens J, Solem C, et al. Pathophysiology and burden of infection in patients with diabetes mellitus and peripheral vascular disease: focus on skin and soft-tissue infections. Clin Microbiol Infect. 2015;21:27-32.

17. Guan W-j, Ni Z-y, Hu Y, Liang W-h, Ou C-q, He J-x, et al. Clinical characteristics of coronavirus disease 2019 in China. New England journal of medicine. 2020;382(18):1708-20.

18. Huang C, Wang Y, Li X, Ren L, Zhao J, Hu Y, et al. Clinical features of patients infected with 2019 novel coronavirus in Wuhan, China. The lancet. 2020;395(10223):497-506.

19. Liu K, Fang Y-Y, Deng Y, Liu W, Wang M-F, Ma J-P, et al. Clinical characteristics of novel coronavirus cases in tertiary hospitals in Hubei Province. Chinese medical journal. 2020.

20. Zhang J-j, Dong X, Cao Y-y, Yuan Y-d, Yang Y-b, Yan Y-q, et al. Clinical characteristics of 140 patients infected with SARS-CoV-2 in Wuhan, China. Allergy. 2020.

21. Walls AC, Park Y-J, Tortorici MA, Wall A, McGuire AT, Veesler D. Structure, function, and antigenicity of the SARS-CoV-2 spike glycoprotein. Cell. 2020.

22. Wrapp D, Wang N, Corbett KS, Goldsmith JA, Hsieh C-L, Abiona O, et al. Cryo-EM structure of the 2019-nCoV spike in the prefusion conformation. Science. 2020;367(6483):1260-3.

23. Xiao F, Tang M, Zheng X, Liu Y, Li X, Shan H. Evidence for gastrointestinal infection of SARS-CoV-2. Gastroenterology. 2020;158(6):1831-3. e3.

24. Zhao D, Yao F, Wang L, Zheng L, Gao Y, Ye J, et al. A comparative study on the clinical features of COVID-19 pneumonia to other pneumonias. Clinical Infectious Diseases. 2020.

25. Mao L, Wang M, Chen S, He Q, Chang J, Hong C, et al. Neurological manifestations of hospitalized patients with COVID-19 in Wuhan, China: a retrospective case series study. 2020.

26. Fodoulian L, Tuberosa J, Rossier D, Landis B, Carleton A, Rodriguez I. SARS-CoV-2 receptor and entry genes are expressed by sustentacular cells in the human olfactory neuroepithelium. bioRxiv. 2020. 
27. Baig AM, Khaleeq A, Ali U, Syeda H. Evidence of the COVID-19 virus targeting the CNS: tissue distribution, host-virus interaction, and proposed neurotropic mechanisms. ACS Chem Neurosci. 2020;11(7):995-8.

28. Sharifi-Razavi A, Karimi N, Rouhani N. COVID-19 and intracerebral haemorrhage: causative or coincidental? New Microbes and New Infections. 2020;35.

29. Poyiadji N, Shahin G, Noujaim D, Stone M, Patel S, Griffith B. COVID-19-associated acute hemorrhagic necrotizing encephalopathy: CT and MRI features. Radiology. 2020:201187.

30. Behzad S, Aghaghazvini L, Radmard AR, Gholamrezanezhad A. Extrapulmonary manifestations of COVID-19: Radiologic and clinical overview. Clinical Imaging. 2020.

31. Zhang W, Du R-H, Li B, Zheng X-S, Yang X-L, Hu B, et al. Molecular and serological investigation of 2019-nCoV infected patients: implication of multiple shedding routes. Emerging microbes infections. 2020;9(1):386-9.

32. Zeng J, Huang J, Pan L. How to balance acute myocardial infarction and COVID-19: the protocols from Sichuan Provincial People's Hospital. Intensive care medicine. 2020:1-3.

33. Jun ISY, Anderson DE, Kang AEZ, Wang L-F, Rao P, Young BE, et al. Assessing viral shedding and infectivity of tears in coronavirus disease 2019 (COVID-19) patients. Ophthalmology. 2020.

\section{Figures}

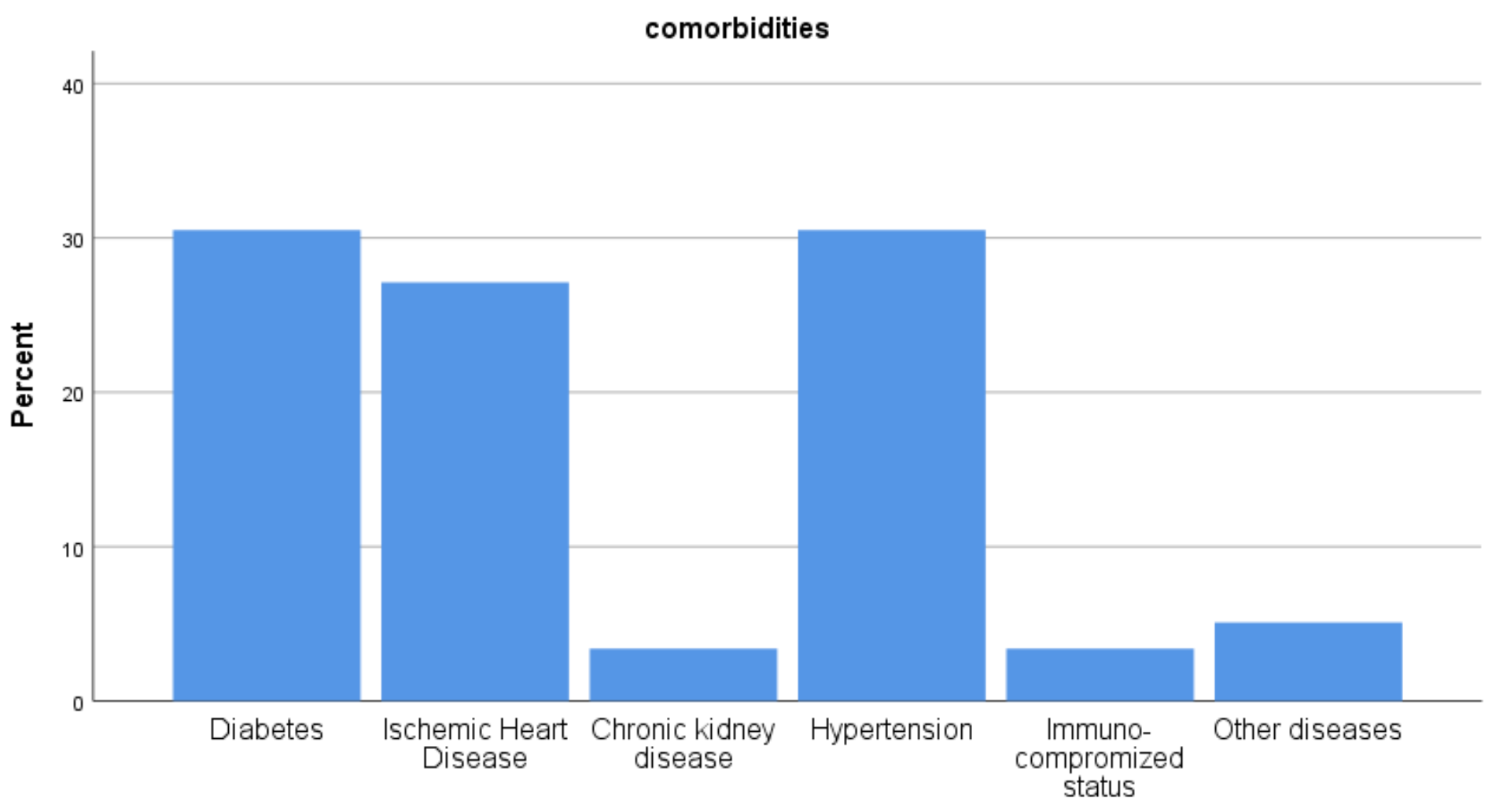

comorbidities

Figure 1 
shows comorbidities of patients on admission.

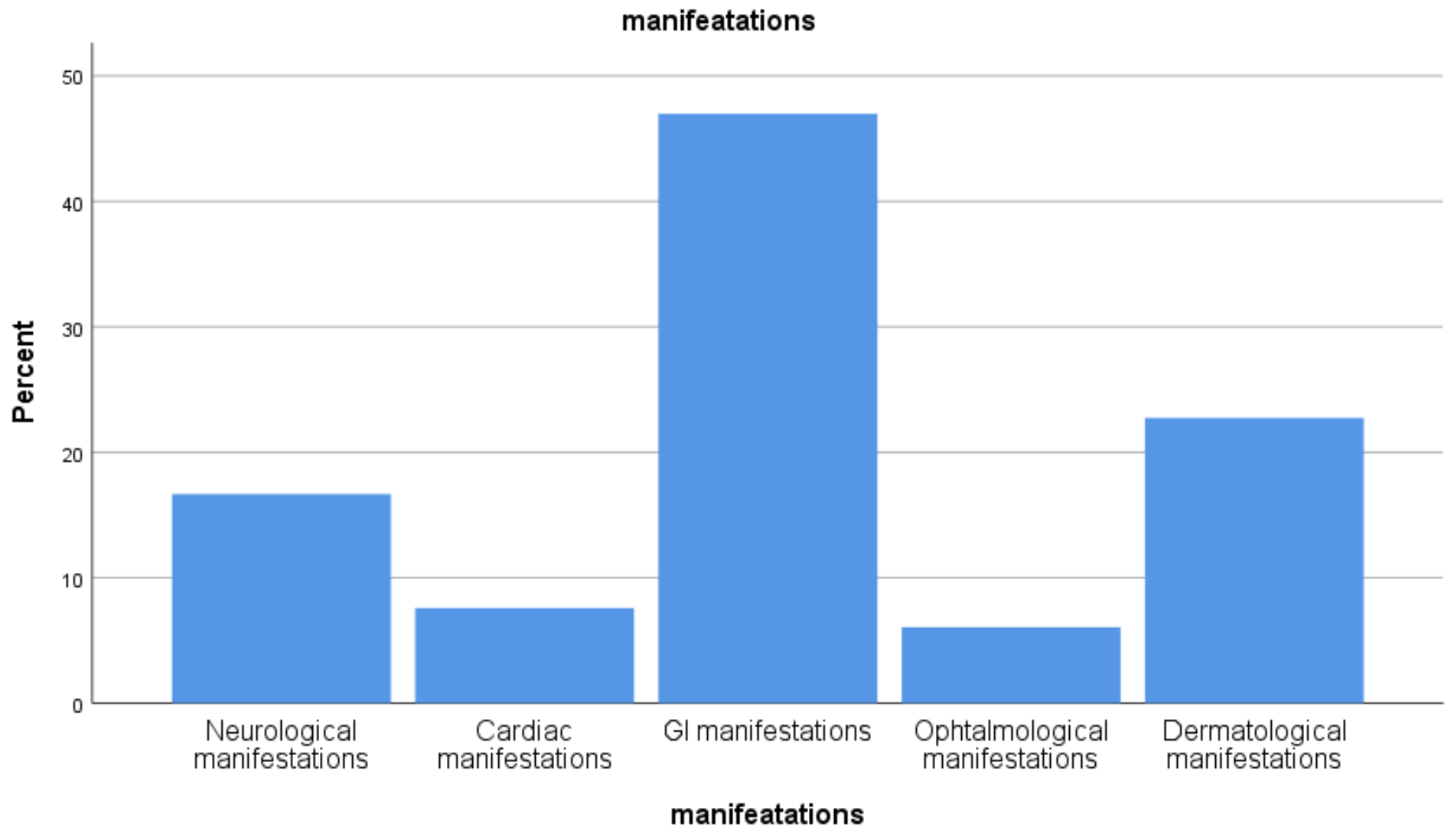

Figure 2

dermatological symptoms such as erythema, macula, papule, morbid form rash, urticarial and patch 